\title{
BACTERIOLOGICAL PROFILE AND ANTIMICROBIAL SUSCEPTIBILITY PATTERNS OF URINE CULTURE ISOLATES FROM PATIENTS IN A TERTIARY CARE CENTRE IN LALITPUR
}

\author{
Manandhar $R^{1^{*}}$, Raghubanshi $B R^{1}$, Mahato $M^{1}$, Neupane $S^{1}$, Lama $R^{1}$
}

\begin{abstract}
Affiliation
1. Department of Microbiology, KIST Medical College and Hospital, Lalitpur, Nepal affliated to Tribhuvan University.
\end{abstract}

\section{ARTICLE INFO}

Received : 23 July, 2019
Accepted : 24 December, 2019
Published : 30 June, 2020

(c) Authors retain copyright and grant the journal right of first publication with the work simultaneously licensed under Creative Commons Attribution License CC - BY 4.0 that allows others to share the work with an acknowledgment of the work's authorship and initial publication in this journal.

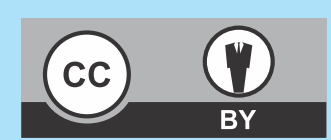

ORA 149

DOI: https://doi.org/10.3126/bjhs.v5i1.29602

* Corresponding Author

Dr. Ruchee Manandhar

Lecturer

Department of Microbiology

KIST Medical College and Hospital, Lalitpur, Nepal

Email: ruchee_manandhar@hotmail.com

ORCID ID: https://orcid.org/0000-0003-3997-5094

\section{Citation}

Manandhar R, Raghubanshi BR, Mahato M, Neupane S, Lama R.Bacteriological Profile and Antimicrobial Susceptibility Patterns of Urine Culture Isolates from Patients in a Tertiary Care Centre in Lalitpur. BJHS 2020;5(1)11:881-885.

\section{ABSTRACT \\ Introduction}

Urinary tract infection (UTI) is a microbial infection of the urinary system involving the urinary tract anywhere from kidney to urethra. It is one of the most common bacterial infections affecting men and women in developing countries with a high rate of morbidity and financial cost.

\section{Objectives}

The objective of our study is to study the prevalence of UTI in patients attending KIST Medical College \& Teaching Hospital (KISTMCTH) and determine the antimicrobial susceptibility pattern of bacteria thus isolated.

\section{Methodology}

A total of 3742 urine samples from patients suspected of urinary tract infections presenting with the history and symptoms suggestive of urinary tract infection, attending various departments of KISTMCTH from April 2017 to April 2018 were studied. Isolates were identified by standard microbiological methods and tested for in vitro antibiotic susceptibility by modified Kirby-Bauer disc diffusion method.

\section{Results}

Pathogenic bacteria were isolated from 646 out of 3742 urine samples (17.26\%). Escherichia coli was the most common bacteria isolated (67.02\%) followed by Klebsiella pneumoniae (14.5\%). Other bacteria isolated were Enterococcus spp, Pseudomonas aeurigenosa, Klebsiella oxytoca, Proteus mirabilis, Satphylococcus aureus, Coagulase negative staphylococcus spp, Proteus vulgaris, Acinetobacter spp, Citrobacter freundii, Citrobacter diversus and Enterobacter spp. The isolated pathogenic bacterias were most resistant to Ampicillin (46.43\%) and least resistant to Imipenem (6.03\%). However, the first line antibiotic the bacterias were least resistant to was cefotaxime (7.12\%).

\section{Conclusions}

Marked resistance has been observed with commonly prescribed antibiotics like Ciprofloxacin and Norfloxacin. Therefore, studies should be conducted routinely to identify the common bacteria causing urinary tract infection and formulate appropriate antibiotic policy.

\section{KEYWORDS}

Antimicrobial resistance, escherichia coli, prevalence, urinary tract infection. 


\section{INTRODUCTION}

Urinary tract infection (UTI) is a microbial infection of the urinary system involving the urinary tract anywhere from kidney to urethra commonly presenting with dysuria, increased urinary frequency, urgency, suprapubic pain and fever. ${ }^{1,2,3}$ The clinical presentation of UTIs however depends on the area of the urinary tract involved, the causative agent, infection severity, age of the patient and the their ability to mount an immune response to it ${ }^{2,3}$ It is one of the most common bacterial infection seen in clinical practice particularly in developing countries with a high rate of morbidity and financial cost. ${ }^{1}$ It affects all age groups and gender in both the community and hospital settings with a marked predilection in females. ${ }^{2}$

Urinary tract infections may be symptomatic or asymptomatic, acute or chronic, and complicated or uncomplicated.

Urinary tract infections are predominantly caused by bacteria and sometimes by viruses and fungi. The most common pathogenic organisms of UTI are Escherichia coli, Staphylococcus saprophyticus, S. aureus, Proteus sp., Klebsiella pneumoniae, Pseudomonas aeruginosa, and enterococci. ${ }^{3}$ A significant number of urinary tract infections are caused by Escherichia coli. ${ }^{1.4}$ Urinary tract infection is considered significant and requires treatment when more than $10^{5}$ colony forming units per $\mathrm{ml}(\mathrm{cfu} / \mathrm{ml})$ of bacteria is present in a clean catch mid stream urine sample. Bacterial count of $10^{4} \mathrm{cfu} / \mathrm{ml}$ is considered significant in specific groups like in children, pregnant women, patients with preexisting kidney diseases or anatomical abnormalities. ${ }^{5}$

Treatment of UTI is often started empirically and therapy is based on information determined from the antimicrobial resistance pattern of the urinary pathogens. ${ }^{6}$ The etiological agents and their susceptibility patterns vary in regions and geographical location that tend to change through time. ${ }^{7}$ Knowledge of the local bacterial etiology and susceptibility patterns not only helps in empirical therapy but also prevents the threat of emerging antimicrobial resistance.

The deadly combination of emerging uropathogens with wide range of virulence factors and widespread antimicrobial resistance threaten the existence of antibiotics as the only effective treatment option available. ${ }^{3}$

The study intends to determine the prevalence of UTI in patients attending KIST Medical College \& Teaching Hospital, find out the antimicrobial susceptibility pattern of bacteria thus isolated.

\section{METHODOLOGY}

This hospital based retrospective study was conducted in Department of Microbiology, KIST Medical College and Teaching hospital, Lalitpur, Nepal. Clearance from the Institutional Research Committee (IRC) KISTMCTH was obtained before conducting the study.

All urine samples from patients suspected of urinary tract infections presenting with the history and symptoms suggestive of urinary tract infection, attending various departments of KIST Medical College and Hospital from April 2017 to April 2018 were studied. Unlabeled or mislabeled urine samples, Urinary catheter tips, Urine samples collected till the rim of the container, Urine samples received beyond 2 hours of collection at room temperature or beyond 24 hours of storage at 4 degrees Celsius were excluded from the study.

All patients were instructed on collection of clean catch mid stream urine sample in order to reduce contamination. The urine samples were properly labeled. Each sample was inoculated into Cystiene Lactose Electrolyte deficient media and incubated at $37^{\circ} \mathrm{C}$ for 24 hours. There is no added benefit to incubating routine urine cultures for 48 hours or beyond as most of the uropathogens grow within 24 hours except for the yeasts. ${ }^{8,9}$ Semi quantitative analysis of the urine sample was done following Kass Criterawhich defined significant bacteriuria as the presence of 100000 or more colonies (CFU) per ml of urine. ${ }^{10}$ Bacterial identification was based on standard microbiological methods. Antimicrobial susceptibility was determined by modified Kirby Bauer disk diffusion method following the criteria designed by the Clinical and Laboratory Standards Institute (CLSI 2011).

The obtained data was entered and analysed in WHONET 5.6 program.

\section{Results}

A total of 3742 urine samples from patients suspected of urinary tract infections attending various departments of KIST Medical College and Hospital were studied. Significant bacteriuria was found and pathogenic bacteria were isolated from 646 out of 3742 urine samples (17.26\%). Fungal growth was obtained in 14 urine samples. There was no growth in 2760 samples. Insignificant growth was found in 26 samples which were not further processed. Majority of urine samples where growth was observed revealed growth of only one type of bacteria. Multiple organisms grew in 296 urine samples (7.91\%) which were not processed further and considered as contamination during sample collection. Repeat sample was then advised.

Out of 646 samples that revealed significant bacteriuria, 470 samples (71\%) belonged to females and 190 samples (29\%) belonged to males.(Figure.1)

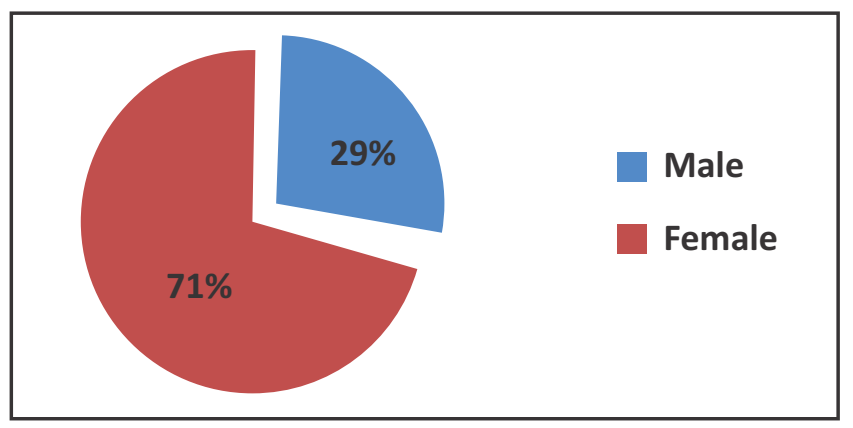

Figure 1: Gender distribution of culture positive isolates

Urine samples from age group ranging 16 years to 30 years revealed maximum culture positivity. (Table.1) 


Table 1: Age distribution of patients with culture positive
isolates
\begin{tabular}{|c|c|}
\hline $\begin{array}{c}\text { Age distribution in } \\
\text { years }\end{array}$ & $\begin{array}{c}\text { Number of culture } \\
\text { positive patients }\end{array}$ \\
\hline $0-15$ & 94 \\
\hline $16-30$ & 231 \\
\hline $31-45$ & 129 \\
\hline $46-60$ & 82 \\
\hline $61-75$ & 66 \\
\hline $76-90$ & 40 \\
\hline Above 90 & 4 \\
\hline TOTAL & 646 \\
\hline
\end{tabular}

Maximum number of growth was found in samples from Department of medicine (28\%). Twelve percentage of samples where significant growth was observed belonged to samples from Pediatric group.

The bacteria that were isolated were Escherichia coli, Klebsiella pneumoniae, Enterococcus spp, Pseudomonas aeurigenosa, Klebsiella oxytoca, Proteus mirabilis, Satphylococcus aureus, Coagulase negative staphylococcus spp, Proteus vulgaris, Acinetobacter spp, Citrobacter freundii, Citrobacter diversus, Enterobacter spp. Escherichia coli was the most common bacteria isolated (67.02\%) followed by Klebsiella pneumoniae (14.5\%). (Table.2)

Table 2: Bacteria Isolated
\begin{tabular}{|c|l|c|c|}
\hline S.No & \multicolumn{1}{|c|}{ Bacteria isolated } & Number & Percentage \\
\hline 1. & Escherichia coli & 433 & $67.02 \%$ \\
\hline 2. & Klebsiella pneumoniae & 94 & $14.5 \%$ \\
\hline 3. & Enterococcus spp & 26 & $4.02 \%$ \\
\hline 4. & Pseudomonas aeurigenosa & 15 & $2.32 \%$ \\
\hline 5. & Klebsiella oxytoca & 13 & $2.01 \%$ \\
\hline
\end{tabular}

Table.3: Antibiogram of Gram positive isolates

\begin{tabular}{|c|c|c|c|c|c|c|c|c|c|c|c|c|c|c|}
\hline \multirow[b]{2}{*}{$\begin{array}{l}\text { S. } \\
\text { No. }\end{array}$} & \multirow[b]{2}{*}{$\begin{array}{l}\text { Name of the } \\
\text { isolates }\end{array}$} & \multirow[b]{2}{*}{$\begin{array}{l}\text { No. of } \\
\text { isolates }\end{array}$} & \multicolumn{12}{|c|}{ Antibiotic resistance pattern } \\
\hline & & & $\begin{array}{c}\text { Amp } \\
(10 \mu g)\end{array}$ & $\begin{array}{l}\text { Cip } \\
(5 \mu \mathrm{g})\end{array}$ & $\begin{array}{c}\mathrm{Na} \\
(30 \mu \mathrm{g})\end{array}$ & $\begin{array}{c}\mathrm{Nx} \\
(10 \mu \mathrm{g})\end{array}$ & $\begin{array}{c}\text { Nit } \\
(300 \mu \mathrm{g})\end{array}$ & $\begin{array}{c}\text { Cot } \\
(25 \mu \mathrm{g})\end{array}$ & $\begin{array}{c}\text { Gen } \\
(10 \mu \mathrm{g})\end{array}$ & $\begin{array}{c}\mathrm{C} \\
(30 \mu \mathrm{g})\end{array}$ & $\begin{array}{c}E \\
15 \mu \mathrm{g})\end{array}$ & $\begin{array}{c}P \\
(2 \mu \mathrm{g})\end{array}$ & $\begin{array}{c}\text { Va } \\
\text { (30ug) }\end{array}$ & $\begin{array}{c}C x \\
(30 \mu g)\end{array}$ \\
\hline 1. & Enterococcus spp & 26 & $\begin{array}{c}6 \\
(37.5)\end{array}$ & $\begin{array}{c}16 \\
(61.53)\end{array}$ & $\begin{array}{c}11 \\
(42.30)\end{array}$ & $\begin{array}{c}16 \\
(61.53)\end{array}$ & $\begin{array}{c}5 \\
(19.23)\end{array}$ & $\begin{array}{c}10 \\
(38.46)\end{array}$ & $\begin{array}{c}11 \\
(42.30)\end{array}$ & $\begin{array}{c}0 \\
(0)\end{array}$ & $\begin{array}{c}0 \\
(0)\end{array}$ & $\begin{array}{c}0 \\
(0)\end{array}$ & $\begin{array}{c}0 \\
(0)\end{array}$ & $\begin{array}{c}0 \\
(0)\end{array}$ \\
\hline 2. & $\begin{array}{l}\text { Staphylococcus } \\
\text { aureus }\end{array}$ & 11 & $\begin{array}{c}4 \\
(36.36)\end{array}$ & $\begin{array}{c}6 \\
(54.54)\end{array}$ & $\begin{array}{c}5 \\
(45.45)\end{array}$ & $\begin{array}{c}7 \\
(63.63)\end{array}$ & $\begin{array}{c}2 \\
(18.18)\end{array}$ & $\begin{array}{c}7 \\
(63.63)\end{array}$ & $\begin{array}{c}2 \\
(18.18)\end{array}$ & - & $\begin{array}{c}0 \\
\text { (0) }\end{array}$ & $\begin{array}{c}0 \\
(0)\end{array}$ & $\begin{array}{c}0 \\
\text { (0) }\end{array}$ & $\begin{array}{c}0 \\
(0)\end{array}$ \\
\hline 3. & $\begin{array}{l}\text { Coagulase egative } \\
\text { Staphylococcus spp }\end{array}$ & 10 & $\begin{array}{c}3 \\
(30)\end{array}$ & $\begin{array}{c}2 \\
(20)\end{array}$ & $\begin{array}{c}0 \\
(0)\end{array}$ & $\begin{array}{c}3 \\
(30)\end{array}$ & $\begin{array}{c}0 \\
(0)\end{array}$ & $\begin{array}{c}3 \\
\text { (30) }\end{array}$ & $\begin{array}{c}0 \\
(0)\end{array}$ & - & $0(0(0)$ & $\begin{array}{c}0 \\
(0)\end{array}$ & $\begin{array}{c}0 \\
(0)\end{array}$ & $\begin{array}{c}0 \\
(0)\end{array}$ \\
\hline & TOTAL & 47 & $\begin{array}{c}13 \\
(27.65)\end{array}$ & $\begin{array}{c}24 \\
(51.06)\end{array}$ & $\begin{array}{c}16 \\
(34.04)\end{array}$ & $\begin{array}{c}26 \\
(55.31)\end{array}$ & $\begin{array}{c}7 \\
(14.89)\end{array}$ & $\begin{array}{c}20 \\
(42.55)\end{array}$ & $\begin{array}{c}13 \\
(27.65)\end{array}$ & $\begin{array}{c}0 \\
(0)\end{array}$ & $\begin{array}{c}0 \\
(0)\end{array}$ & $\begin{array}{c}0 \\
(0)\end{array}$ & $\begin{array}{c}0 \\
\text { (0) }\end{array}$ & $\begin{array}{c}0 \\
(0)\end{array}$ \\
\hline
\end{tabular}

Numbers in parenthesis indicate percentage

\begin{tabular}{|c|l|c|c|}
\hline 6. & Proteus mirabilis & 12 & $1.8 \%$ \\
\hline 7 & Staphylococcus aureus & 11 & $1.7 \%$ \\
\hline 8. & $\begin{array}{l}\text { Coagulase negative } \\
\text { staphylocoocus spp (CoNS) }\end{array}$ & 10 & $1.5 \%$ \\
\hline 9. & Proteus vulgaris & 8 & $1.2 \%$ \\
\hline 10. & Acinetobacter spp & 7 & $1.08 \%$ \\
\hline 11. & Citrobacter freundii & 8 & $1.23 \%$ \\
\hline 12. & Citrobacter diversus & 4 & $0.61 \%$ \\
\hline 13. & Enterobacter spp & 5 & $0.77 \%$ \\
\hline & TOTAL & $\mathbf{6 4 6}$ & $\mathbf{1 0 0 \%}$ \\
\hline
\end{tabular}

Candida spp was isolated from 14 samples. Antibiotic sensitivity test was performed on all bacterial isolates.

Specimens were not processed for presence of anaerobic bacteria, viruses or parasites.

Antimicrobial susceptibility test revealed that the isolated bacterias were most resistant to ampicillin (46.43\%). The isolated bacterias were least resistant to Imipenem and Amoxicillin clavulinic acid showing resistance of $6.03 \%$ and $6.81 \%$ respectively. However, the first line antibiotic the bacterias were least resistant to was cefotaxime (7.12\%). Among the Gram positive organism, Nitrofurantoin was found to be the most sensitive drug (85.11\%). Among the Gram negative bacteria, Imipenem was found to be the most sensitive drug (93.9\%) followed by cefotaxime (93\%). Escherichia coli was found to be most sensitive to Imipenem (96.07\%) and amoxicillin clavulinic acid (93.31\%). (Table.3 and Table.4)

\begin{tabular}{|c|c|c|c|c|c|c|c|c|c|c|c|c|c|c|c|c|}
\hline & \multirow{2}{*}{$\begin{array}{l}\text { S. } \\
\text { No. }\end{array}$} & \multirow{2}{*}{$\begin{array}{l}\text { Name of the } \\
\text { isolates }\end{array}$} & \multirow{2}{*}{$\begin{array}{c}\text { Number } \\
\text { of } \\
\text { isolates }\end{array}$} & \multicolumn{13}{|c|}{ Antibiotic resistance pattern } \\
\hline & & & & 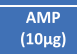 & 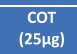 & $\begin{array}{c}\mathrm{NX} \\
\text { (10mg) }\end{array}$ & $\begin{array}{c}\mathrm{NIT} \\
(300 \mu \mathrm{g})\end{array}$ & $\begin{array}{c}\text { NA } \\
\text { (30ug) }\end{array}$ & 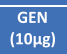 & 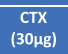 & $\begin{array}{c}\mathrm{CIP} \\
(5 \mu \mathrm{g})\end{array}$ & $\begin{array}{c}\text { CTR } \\
\text { (30mg) }\end{array}$ & $\begin{array}{r}\text { IPM } \\
(10 \mu \mathrm{g})\end{array}$ & $\begin{array}{c}\mathrm{AK} \\
(10 \mathrm{\mu g})\end{array}$ & 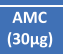 & $\begin{array}{c}\text { Of } \\
\text { (5ug) }\end{array}$ \\
\hline & 1. & E.coli & 433 & $\begin{array}{r}208 \\
(48.03)\end{array}$ & $\begin{array}{r}181 \\
(41.80)\end{array}$ & $\begin{array}{c}181 \\
(41.80)\end{array}$ & $\begin{array}{c}36 \\
(8.31)\end{array}$ & $\begin{array}{l}128 \\
(29.5)\end{array}$ & $\begin{array}{c}50 \\
(11.54)\end{array}$ & $\begin{array}{c}30 \\
(6.92)\end{array}$ & $\begin{array}{c}183 \\
(42.26)\end{array}$ & $\begin{array}{c}143 \\
(33.02)\end{array}$ & $\begin{array}{c}17 \\
(3.9)\end{array}$ & $\begin{array}{c}34 \\
(7.85)\end{array}$ & $\begin{array}{c}29 \\
(6.69)\end{array}$ & $\begin{array}{c}31 \\
(7.15)\end{array}$ \\
\hline & 2. & Kleb. pneumoniae & 94 & $\begin{array}{c}52 \\
(55.3)\end{array}$ & $\begin{array}{c}36 \\
(38.2)\end{array}$ & $\begin{array}{c}38 \\
(40.4)\end{array}$ & $\begin{array}{l}47 \\
(50)\end{array}$ & $\begin{array}{c}21 \\
(22.3)\end{array}$ & $\begin{array}{c}30 \\
(31.9)\end{array}$ & $\begin{array}{c}7 \\
(7.44)\end{array}$ & $\begin{array}{c}33 \\
(35.1)\end{array}$ & $\begin{array}{c}36 \\
(38.2)\end{array}$ & $\begin{array}{c}14 \\
(14.8)\end{array}$ & $\begin{array}{c}23 \\
(24.4)\end{array}$ & $\begin{array}{c}6 \\
(6.3)\end{array}$ & $\begin{array}{c}9 \\
(9.5)\end{array}$ \\
\hline & 3. & $\begin{array}{l}\text { Pseudomonas } \\
\text { aeurigenosa }\end{array}$ & 15 & $\begin{array}{c}5 \\
(33.3)\end{array}$ & $\begin{array}{c}8 \\
(53.3)\end{array}$ & $\begin{array}{c}9 \\
(60)\end{array}$ & $\begin{array}{c}10 \\
(66.6)\end{array}$ & $\begin{array}{c}4 \\
(26.6)\end{array}$ & $\begin{array}{c}9 \\
(60)\end{array}$ & $\begin{array}{c}1 \\
(6.6) \\
\end{array}$ & $\begin{array}{c}8 \\
(53.3) \\
\end{array}$ & $\begin{array}{c}6 \\
(40)\end{array}$ & $\begin{array}{c}3 \\
(20)\end{array}$ & $\begin{array}{c}8 \\
(53.3)\end{array}$ & $\begin{array}{c}0 \\
\text { (0) }\end{array}$ & $\begin{array}{c}5 \\
(33.3)\end{array}$ \\
\hline & 4. & Klebsiella oxytoca & 13 & $\begin{array}{c}3 \\
(23)\end{array}$ & $\begin{array}{l}17 \\
(7.6)\end{array}$ & $\begin{array}{c}2 \\
15.3\end{array}$ & $\begin{array}{c}1 \\
(7.6)\end{array}$ & $\begin{array}{c}3 \\
(23)\end{array}$ & $\begin{array}{c}0 \\
(0)\end{array}$ & $\begin{array}{c}1 \\
(7.6)\end{array}$ & $\begin{array}{c}2 \\
(15.3)\end{array}$ & $\begin{array}{c}2 \\
2 \\
(15.3)\end{array}$ & $\begin{array}{c}1 \\
(7.6)\end{array}$ & $\begin{array}{c}0 \\
(0)\end{array}$ & $\begin{array}{c}1 \\
(7.6)\end{array}$ & $\begin{array}{c}0 \\
(0)\end{array}$ \\
\hline & 5. & Proteus mirabilis & 12 & $\begin{array}{c}3 \\
(25)\end{array}$ & $\begin{array}{c}7 \\
58.3\end{array}$ & $\begin{array}{c}4 \\
33.3\end{array}$ & $\begin{array}{c}9 \\
(75)\end{array}$ & $\begin{array}{c}6 \\
(50)\end{array}$ & $\begin{array}{c}3 \\
\text { (25) }\end{array}$ & $\begin{array}{c}1 \\
(8.3)\end{array}$ & $\begin{array}{c}4 \\
(33.3)\end{array}$ & $\begin{array}{c}4 \\
(33.3)\end{array}$ & $\begin{array}{c}0 \\
(0)\end{array}$ & $\begin{array}{c}2 \\
2 \\
(16.6)\end{array}$ & $\begin{array}{c}2 \\
2 \\
(16.6)\end{array}$ & $\begin{array}{l}0 \\
\text { (0) }\end{array}$ \\
\hline & 6. & Proteus vulgaris & 8 & $\begin{array}{c}6 \\
65)\end{array}$ & $\begin{array}{c}5 \\
(62.5)\end{array}$ & $\begin{array}{c}3 \\
(37.5)\end{array}$ & $\begin{array}{c}3 \\
(37.5)\end{array}$ & $\begin{array}{c}2 \\
25)\end{array}$ & $\begin{array}{c}1 \\
(12.5)\end{array}$ & $\begin{array}{l}0 \\
0\end{array}$ & $\begin{array}{c}2 \\
(25)\end{array}$ & $\begin{array}{c}0 \\
(0)\end{array}$ & $\begin{array}{l}0 \\
\text { (0) }\end{array}$ & $\begin{array}{c}2 \\
(25)\end{array}$ & $\begin{array}{c}0 \\
0 \\
(0)\end{array}$ & $\begin{array}{c}0 \\
0 \\
(0)\end{array}$ \\
\hline & 7. & Citrobacter freundii & 8 & $\begin{array}{c}3 \\
(37.5)\end{array}$ & $\begin{array}{c}3 \\
(37.5)\end{array}$ & $\begin{array}{c}2 \\
(25)\end{array}$ & $\begin{array}{c}4 \\
(50)\end{array}$ & $\begin{array}{l}0 \\
0 \\
(0)\end{array}$ & $\begin{array}{c}0 \\
0 \\
(0)\end{array}$ & $\begin{array}{l}0 \\
0 \\
(0)\end{array}$ & $\begin{array}{c}2 \\
(25)\end{array}$ & $\begin{array}{c}2 \\
25)\end{array}$ & $\begin{array}{c}1 \\
(12.5)\end{array}$ & $\begin{array}{c}127 \\
(12.5)\end{array}$ & $\begin{array}{c}2 \\
(25)\end{array}$ & $\begin{array}{c}1 \\
12.5)\end{array}$ \\
\hline & 8. & \begin{tabular}{|l} 
Citrobacter diversus \\
\end{tabular} & 4 & $\begin{array}{c}2 \\
(50)\end{array}$ & $\begin{array}{c}0 \\
(0)\end{array}$ & $\begin{array}{c}0 \\
(0)\end{array}$ & $\begin{array}{c}0 \\
\text { (0) }\end{array}$ & $\begin{array}{c}0 \\
\text { (0) }\end{array}$ & $\begin{array}{c}0 \\
(0)\end{array}$ & $\begin{array}{c}1 \\
(25)\end{array}$ & $\begin{array}{c}0 \\
(0)\end{array}$ & $\begin{array}{c}1 \\
(25)\end{array}$ & $\begin{array}{c}0 \\
(0)\end{array}$ & $\begin{array}{c}0 \\
(0)\end{array}$ & $\begin{array}{c}0 \\
(0)\end{array}$ & $\begin{array}{c}0 \\
\text { (0) }\end{array}$ \\
\hline & 9. & Acinetobacter spp & 7 & $\begin{array}{c}2 \\
(28)\end{array}$ & $\begin{array}{c}3 \\
(42.8)\end{array}$ & $\begin{array}{c}5 \\
(71.4)\end{array}$ & $\begin{array}{c}5 \\
(71.4)\end{array}$ & $\begin{array}{c}2 \\
28.5)\end{array}$ & $\begin{array}{c}3 \\
(42.85)\end{array}$ & $\begin{array}{c}1 \\
(14.28)\end{array}$ & $\begin{array}{c}4 \\
(57.14)\end{array}$ & $\begin{array}{c}2 \\
(28.57)\end{array}$ & $\begin{array}{c}0 \\
(0)\end{array}$ & $\begin{array}{c}4 \\
(57.14)\end{array}$ & $\begin{array}{c}1 \\
(14.2)\end{array}$ & $\begin{array}{c}0 \\
\text { (0) }\end{array}$ \\
\hline & 10. & \begin{tabular}{|l} 
Enterobacter spp \\
\end{tabular} & 5 & $\begin{array}{c}3 \\
(66)\end{array}$ & $\begin{array}{c}1 \\
(20)\end{array}$ & $\begin{array}{c}1 \\
(20)\end{array}$ & $\begin{array}{l}0 \\
(0)\end{array}$ & $\begin{array}{c}3 \\
(60) \\
\end{array}$ & $\begin{array}{c}0 \\
0 \\
(0)\end{array}$ & $\begin{array}{c}1+3 \\
0 \\
(0)\end{array}$ & $\begin{array}{c}0 \\
0 \\
(0)\end{array}$ & $\begin{array}{c}0 \\
0 \\
(0)\end{array}$ & $\begin{array}{c}1 \\
(20)\end{array}$ & $\begin{array}{c}0 \\
0 \\
(0)\end{array}$ & $\begin{array}{c}1 \\
(20)\end{array}$ & $\begin{array}{c}1 \\
(20)\end{array}$ \\
\hline & & TOTAL & 599 & $\begin{array}{l}287 \\
(47.9)\end{array}$ & $\begin{array}{c}261 \\
(43.5)\end{array}$ & $\begin{array}{l}245 \\
(40.9)\end{array}$ & $\begin{array}{c}115 \\
(19.1)\end{array}$ & $\begin{array}{c}169 \\
(28.2)\end{array}$ & $\begin{array}{l}96 \\
(16.02)\end{array}$ & $\begin{array}{l}42 \\
(7)\end{array}$ & $\begin{array}{c}236 \\
(39.3)\end{array}$ & $\begin{array}{c}196 \\
(32.7)\end{array}$ & $\begin{array}{c}37 \\
(6.1)\end{array}$ & $\begin{array}{c}74 \\
(12.3)\end{array}$ & $\begin{array}{c}42 \\
(7.01)\end{array}$ & $\begin{array}{c}47 \\
(7.8)\end{array}$ \\
\hline \multirow{2}{*}{883} & \multicolumn{16}{|c|}{ Birat Journal of Health Sciences } \\
\hline & Vol.5/ & o.1/Issue 11 / Ja & April, 20 & & & ISSN: 2 & $2-2758$ & int) 25 & $2804(C$ & line) & & & & & & \\
\hline
\end{tabular}


Numbers in parenthesis indicate percentage

Order for resistance pattern was Ampicillin > Norfloxacin > Cotrimoxazole $>$ Ciprofloxacin $>$ Ceftriaxone $>$ Nalidixic acid $>$ Nitrofurantoin > Gentamicin > Amikacin > Ceftazidime > Cefotaxime > Amoxycillin clavulinic acid > Imipenem. (Figure.2)

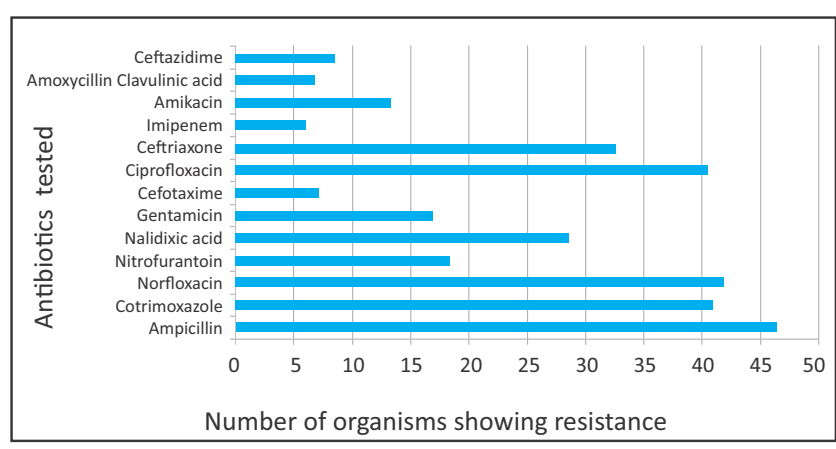

\section{DISCUSSION}

In developing countries urinary tract infection (UTI) is one of the most commonly diagnosed disease among the patients seeking medical treatment with frequency of 180 per $10,000 .{ }^{11}$ Our study shows the prevalence rate of $17.26 \%$ which was similar to studies conducted in Kathmandu medical college \& hospital and B\&B hospital, Lalitpur which reported a prevalence rate of $13.8 \%$ and $25.24 \%$ respectively. ${ }^{11,12} \mathrm{~A}$ study conducted in Western Nepal shows a higher prevalence rate of $43.25 \% .{ }^{13}$ However, prevalence was found to be $39.69 \%$ in a rural community of Nigeria. ${ }^{14}$ This variations in the prevalence of UTIs may result from different environmental conditions, host factors, socioeconomic status, hygiene practices and execution of healthcare and education programmes within communities of different geographical areas.

As shown in Figure.1, prevalence rate was found to be higher in females $(71 \%)$ than in males $(29 \%)$ were similar to studies conducted in Nepal and other countries. ${ }^{1-3,11-14}$ This might be because of the short urethra and close proximity of the urethra to the perianal region and the lack of secretion produced from prostate present in males which has bactericidal property.

The organism isolated from this study as depicited in Table. 2 is similar to the studies conducted in different parts of the world. ${ }^{1-3,5-7,11-14}$ In our study, Escherichia coli was the most common bacteria isolated (67.02\%) followed by Klebsiella pneumoniae (14.5\%) which was similar to the study conducted in Kathmandu Medical College \& Hospital and Nepal medical college \& Hospital in Kathmandu and in a multicenter study conducted in India. ${ }^{11,15,16}$ The organisms were most resistant to Ampicillin( 46.43\%) as shown in Figure. 2 which was similar to studies conducted by Russel Kabir et al, Rijal et al and Thapa et al in Nepal. $(3,5,6)$ In contrast to our study, organisms were most sensitive to ciprofloxacin $(63 \%)$ in a study conducted in Kathmandu
Medical College. However, in our study ciprofloxacin was sensitive in only $59.45 \%$. Escherichia coli was found to be most sensitive to Imipenem (96.07\%) which was similar to a study conducted in Punjab. In a study conducted by Kibret et al, Nitrofurantoin, Gentamicin and ciprofloxacin were considered as appropriate antimicrobials for empirical treatment of UTI in the area of Ethiopia which was in contrast to our study. ${ }^{17}$

\section{CONCLUSION}

Urinary tract infection is the most common bacterial infection seen in clinical practice. Escherichia coli remains the most common pathogen causing UTI. Female patients in reproductive age groups are more prone to develop UTI. The bacteria isolated were most sensitive to Imipenem \& amoxicillin clavulinic acid. However, the first line antibiotic that the bacteria were most sensitive remains cefotaxime. Marked resistance has been observed with commonly prescribed antibiotics like Ciprofloxacin and Norfloxacin. Therefore, studies should be conducted routinely to identify the common bacteria causing UTI and formulate appropriate antibiotic policy.

\section{RECOMMENDATIONS}

Surveillance programs should be conducted regularly to identify the prevalent organisms and their antibiotic sensitivity pattern to formulate antibiotic policy that can be implemented for making proper guidelines regarding empirical therapy.

\section{LIMITATIONS OF THE STUDY}

- Only aerobic bacteriological assessment was carried out. Routine urine examination was not included to justify urine culture as it can be a prospective study in itself.

- Extended Spectrum Beta Lactamase(ESBL) producing bacteria were not identified.

- Molecular identification of the isolates was not included in the study to substantiate epidemiological relationship.

\section{ACKNOWLEDGEMENTS}

We are grateful to Microbiology laboratory staffs at KISTMCTH for their help and support.

\section{CONFLICT OF INTEREST}

None

FINANCIAL DISCLOSURE 


\section{REFERENCES}

1. Barber E. Amelia , Norton J. Paul, Spivak M.Adam, and Mulvey A.Matthew. Urinary Tract Infections: Current and Emerging Management Strategies Clin Infect Dis 2013 Sep 1; 57(5): 719-724. DOI : $10.1093 /$ cid/cit284

2. Ahmed Al-Badr and Ghadeer Al-Shaikh. Recurrent Urinary Tract Infections Management in Women A review. Sultan Qaboos Univ Med J. 2013 Aug; 13(3): 359-367. PMID: 23984019

3. Flores-Mireles AL, Walker JN, Caparon M, Hultgren SJ. Urinary tract infections: epidemiology, mechanisms of infection and treatment options. Nat Rev Microbiol. 2015;13(5):269-284. DOI: 10.1038/ nrmicro3432

4. Almulhim AS, Aldayyen A, Yenina K, Chiappini A, Khan TM. Optimization of antibiotic selection in the emergency department for urine culture follow ups, a retrospective pre-post intervention study: clinical pharmacist efforts. J Pharm Policy Pract. 2019;12:8. DOI: 10.1186/s40545-019-0168-z

5. Kline KA, Lewis AL. Gram-Positive Uropathogens, Polymicrobial Urinary Tract Infection, and the Emerging Microbiota of the Urinary Tract. Microbiol Spectr. 2016;4(2):10. DOI: 10.1128/ microbiolspec. UTI-0012-2012.

6. Beahm NP, Nicolle LE, Bursey A, Smyth DJ, Tsuyuki RT. The assessment and management of urinary tract infections in adults: Guidelines for pharmacists.Can Pharm J (Ott). 2017;150(5):298-305. DOI: 10.1177/ 1715163517723036

7. Derbie A, Hailu D, Mekonnen D, Abera B, Yitayew G. Antibiogram profile of uropathogens isolated at Bahir Dar Regional Health Research Laboratory Centre, Northwest Ethiopia. Pan Afr Med J. 2017;26:134 DOI:10.11604/pamj.2017.26.134.7827.

8. Wilson L. Michael, Gaido Loretta. Laboratory Diagnosis of Urinary Tract Infections in Adult Patients, Clinical Infectious Diseases, 2004;38(8) : 1150-1158. https://doi.org/10.1086/383029
9. Noorbakhsh, Mir H. "1998. Urine Culture Incubation Time: One vs. 2 Days!." Open Forum Infectious Diseases.2018;5(1):S581 PMID: PMC6253415

10. Kass EH. Asymptomatic infections of the urinary tract. Trans Assoc Am Physicians. 1956;69:56-64. [PubMed] [Google Scholar] PMID: 13380946

11. Pradhan B, Pradhan SB. Prevalence of Urinary Tract Infection and Antibiotic Susceptibility Pattern to Urinary Pathogens in Kathmandu Medical College and Teaching Hospital, Duwakot BJHS 2017; 2 (1) 2 : 134-137. DOI: https://doi.org/10.3126/bjhs.v2i1.17290

12. Yadav Prasad Joshi, Shreejeet Shrestha, Russell Kabir, Anita Thapa, Parbati Upreti, Sanjit Shrestha Urinary tract infections and antibiotic susceptibility among the patients attending B \& D hospital of Lalitpur, Nepal. Asian Journal of Medical Sciences Vol 7, No 5 (2016) DOI: https://doi.org/10.3126/ajms.v7i5.14908

13. P Thapa, K Parajuli , A Poudel, A Thapa, B Manandhar, D Laudari, HB Malla, R Katiwada. Causative agentsand susceptibility of antimicrobials among suspected females with urinary tract infections IN in tertiary care hospitals of Western Nepal Journal of Chitwan Medical College 2013; 3(4): 16-19

14. Bankole Henry Oladeinde, MSc., AIMLS., Richard Omoregie, MPhil., FIMLS., Mitsan Olley, MSc., AIMLS, and Joshua A. Anunibe, MSc., AIMLS. Urinary tract infection in a rural community of Nigeria. N Am J Med Sci. 2011 Feb; 3(2): 75-77. PMID: 22540069

15. Rijal A, Ghimire G, Gautam K, Barakoti A. Antibiotic Susceptibility of Organisms Causing Urinary Tract Infection in Patients Presenting to a Teaching Hospital. J Nepal Health Res Counc 2012 Jan;10(20):24-7.

16. Kothari Atul and Sagar Vishal Antibiotic resistance in pathogens causing community-acquired urinary tract infections in India: a multicenter study JIDC Vol 2 No 05: October 2008 PMID: 19745502

17. Kibret $M$, Abera B. Prevalence and antibiogram of bacterial isolates from urinary tract infections at Dessie Health Research Laboratory, Ethiopia. Asian Pac J Trop Biomed. 2014;4(2):164-168. DOI: 10.1016/ S2221-1691(14)60226-4. 